\section{Die Akademie für Fort- und Weiterbildung in der Radiologie erweitert ihr Serviceangebot für Veranstalter von Fortbildungen}

Ab dem 1. Januar 2014 bieten wir allen Organisatoren von Fortbildungsveranstaltungen, die ein radiologisches Schwerpunktthema haben oder für Radiologen interessant sein könnten zusätzliche Serviceleistungen an. Unser Ziel ist es, Sie bei der Bewerbung, Vorbereitung und Evaluation Ihrer Veranstaltung optimal zu unterstützen. Im Folgenden stellen wir Ihviceleistungen vor. Sicherlich finden Sie zukünftig für Ihre Veranstaltung das passende Angebot.

\section{- Veröffentlichung Ihrer Veranstal- tung im Online-Veranstaltungska- lender als Einzelleistung \\ - 100.-€ für wissenschaftliche / kli- nisch organisierte Veranstaltungen \\ > 400.-€ für industrielle Veranstaltun- gen} nen unsere bewährten und neuen Ser-
Mit dem Eintrag Ihrer Veranstaltung in unseren Online-Veranstaltungskalender erreichen Sie eine Vielzahl potentieller Teilnehmer und bewerben Ihre Veranstaltung kostengünstig. Beantragen Sie die Veröffentlichung Ihrer Veranstaltung über unser Online-Formular (www.drgakademie.de - Veranstalter - Veranstaltung) und die Daten werden zeitnah in unseren Veranstaltungskalender aufgenommen.

\section{- Veröffentlichung Ihrer Veranstal-} tung im Online-Veranstaltungskalender und Bewerbung in RÖFO, Der Radiologe und im Akademie-Newsletter

> 200.-€ für wissenschaftliche / klinisch organisierte Veranstaltungen

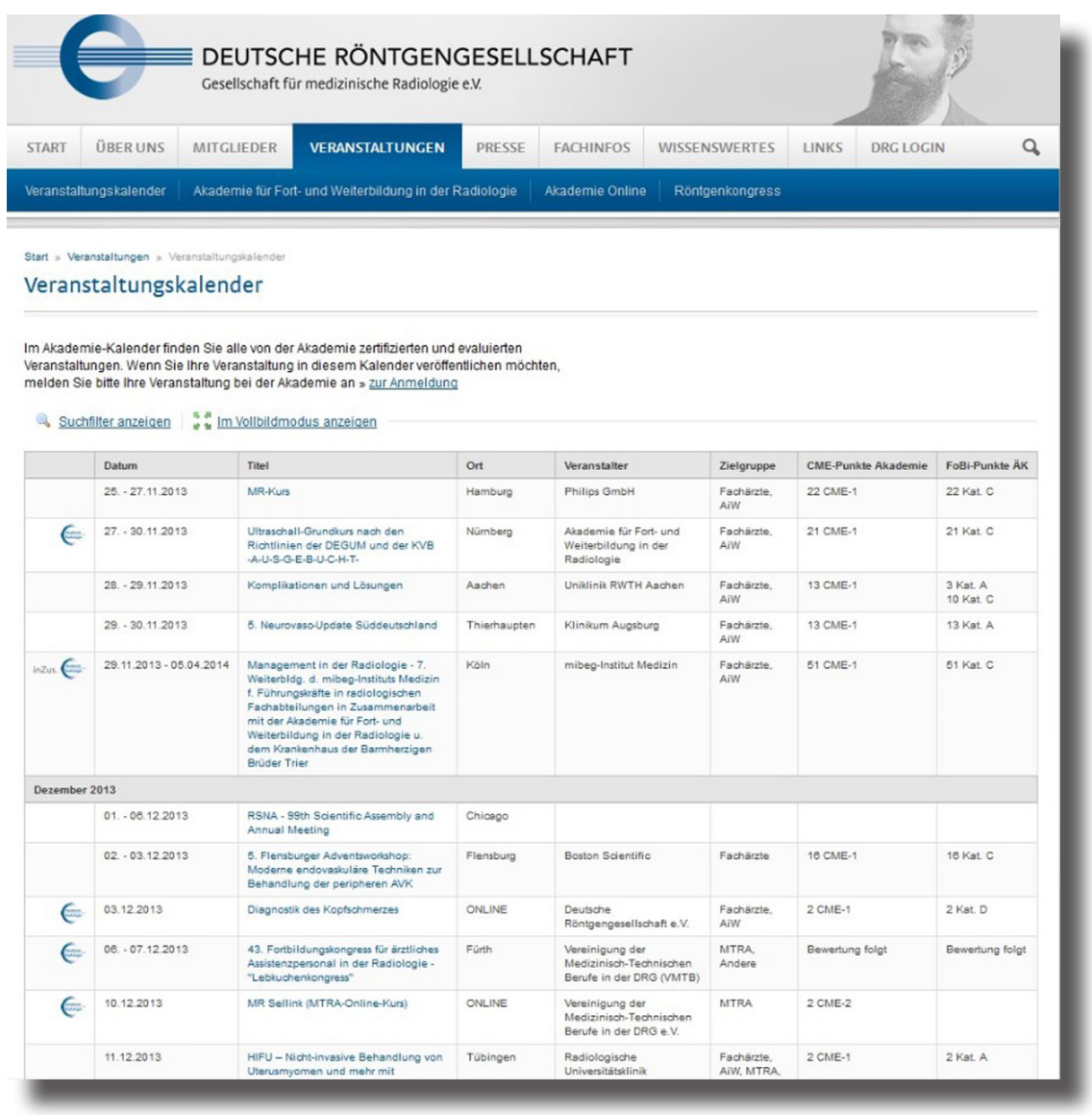

-600.-€ für industrielle Veranstaltungen

Sie möchten Ihrer Veranstaltung durch aktives Bewerben zu noch mehr Aufmerksamkeit verhelfen (Voraussetzung ist hier allerdings, dass Sie Ihre Veranstaltung von der zuständigen Ärztekammer zertifiziren lassen). Dann ist dieses Angebot für Sie richtig. Ihre Veranstaltung wird zusätzlich in den Veranstaltungskalendern der Fachzeitschriften „Fortschritte auf dem Gebiet der Röntgenstrahlen und der bildgebenden Verfahren - RÖFO“und „Der Radiologe“ und im Newsletter der Akademie für Fort- und Weiterbildung in der Radiologie angezeigt. Den Newsletter versenden wir 14-tägig an über 8000 Interessenten (Mitglieder der DRG, VMTB u.a.). Er verweist auf Veranstaltungen der kommenden 12 Monate.

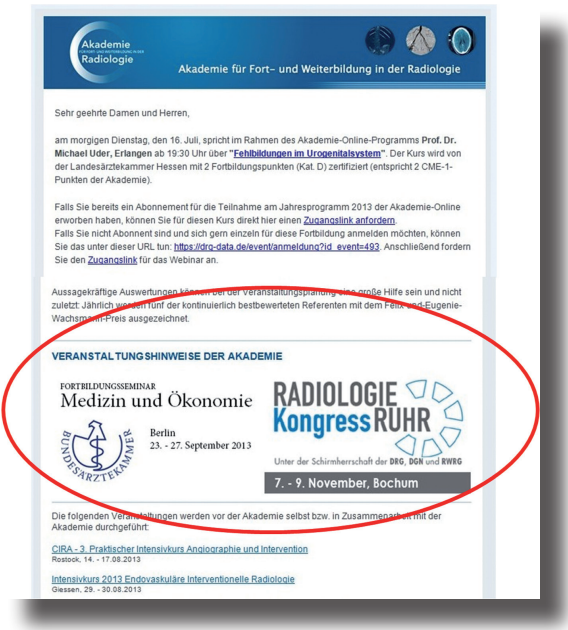

- Veröffentlichung Ihrer Veranstaltung im Online-Veranstaltungskalender und Bewerbung in RÖFO, Der Radiologe und im Akademie-Newsletter inkl. zweimaliger Platzierung Ihres Veranstaltungslogos

- 300.-€ für wissenschaftliche / klinisch organisierte Veranstaltungen - 800.-€ für industrielle Veranstaltungen

Sie möchten sich zusätzlich zur Anzeige im Online-Veranstaltungskalender der Akademie für Fort- und Weiterbildung in der Radiologie und in den Fachzeitschriften „Fortschritte auf dem Gebiet der Röntgenstrahlen und der bildgebenden Verfahren - RÖFO“ und „Der Radiologe“ einen Premium-Platz im Newsletter der 
Akademie sichern? Übersenden Sie uns Ihr Veranstaltungslogo! Ihre Veranstaltung, an exponierter Stelle im Newsletter platziert, wird damit optisch deutlich hervorgehoben. Der Newsletter erscheint 14-tägig, zweimal wird Ihr Veranstaltungslogo veröffentlicht, natürlich in Absprache mit Ihnen.

\footnotetext{
- Aufnahme in das CME-Programm der Akademie - Veröffentlichung Ihrer Veranstaltung im Online-Veranstaltungskalender, Bewerbung in RÖFO und Der Radiologe, Zertifizierung und Evaluation

- 100.- € Grundgebühr für wissenschaftliche / klinisch organisierte Veranstaltungen

> 400.-€ Grundgebühr für industrielle Veranstaltungen

- 200.- € Grundgebühr für wissenschaftliche / klinisch organisierte Veranstaltungen in Zusammenarbeit mit der Akademie

- zusätzliche Bewerbung im Akademie-Newsletter (Versand 14-tägig)
}

- Kennzeichnung im Veranstaltungskalender mit unserem Qualitätssiegel

- Antragstellung bei der Ärztekammer und Erstellung der Vorlage für die Teilnahmebescheinigung auf Wunsch

- Zuzüglich 20.- € pro CME-Punkt, 2,50€ pro Teilnehmer

Die Qualität Ihrer Veranstaltung, aufbauend auf der Befähigung Ihrer Referenten, hat für Sie oberste Priorität. Die Evaluation der Akademie für Fort- und Weiterbildung in der Radiologie steht für die Sicherung und kontinuierliche Verbesserung dieser Qualität. Mit der Evaluation geben Sie Ihren Referenten einen besonderen Anreiz, Gewinner des Wachsmannpreises zu werden, der jährlich auf dem Röntgenkongress an die bestbewerteten Referenten vergeben wird.

Zusätzlich zu den oben genannten Angeboten besteht die Möglichkeit, eine $\mathrm{Zu}$ sammenarbeit mit der Akademie zu beantragen. Diese Vereinbarung beinhaltet die
Veröffentlichung Ihrer Veranstaltung im Veranstaltungskalender unter besonderer Hervorhebung durch das Qualitätssiegel der Akademie für Fort- und Weiterbildung in der Radiologie. Darüber hinaus übernehmen wir auf Wunsch die Antragstellung bei der zuständigen Ärztekammer für Sie und stellen Ihnen gerne eine Vorlage für Ihre Teilnahmebescheinigungen zur Verfügung. Sie platzieren unser Logo auf Ihren Werbematerialien, wie z.B. Programmen, Plakaten, Flyern und allen weiteren Veröffentlichungen sowie auf den ggf. selbst erstellten Teilnahmebescheinigungen.

Gerne berät Sie Frau Sabine Strauß (Tel.:030-91607033, Email: strauss@drg. de ) bei der Auswahl eines unserer Angebote, um die für Ihre Veranstaltung passende Serviceleistung zu finden. 